\title{
Investigation of Algorithms for VolP Signaling
}

\author{
Todorka Georgieva $^{1}$, Ekaterina Dimitrova ${ }^{2}$ and Slava Yordanova ${ }^{3}$ \\ 1. Telecommunication Department, Technical University of Varna, Studentska 1, 9010 Varna, Bulgaria \\ 2. Department of Electronics and Microelectronics, Technical University of Varna, Studentska 1, Bulgaria \\ 3. Department of Computer Science and Engineering, Technical University of Varna, Studentska 1, Bulgaria
}

\begin{abstract}
This paper discusses issues regarding the operation of algorithms for VoIP (voice over internet protocol) signaling. The resulting data include jitter, packet delays, delays related to signalization, distribution of packets in protocols and relative assessments of QoS (quality of service) and MOS (mean opinion score).
\end{abstract}

Key words: Codec, packet, signalization, VoIP.

\section{Introduction}

The main objective of this paper is to solve problems with the implementation of voice applications over wireless networks with packet switching, the ability to overcome them and to optimize this type of communication. The extended IP structures, including wireless, provide large opportunities for the development of VoIP (voice over internet protocol) technologies. The paper concerns the methods of improving the quality of service, which most closely affect the ultimate user. Concrete results are obtained proving the future application of these technologies.

Conducted studies show that the problems associated with the range of wireless networks and their security cannot be a real obstacle to the implementation of Vo802.11.

The propounded thesis demonstrates the broad possibilities for the implementation of VoIP over 802.11 and the provision of new services to existing ones [1].

One of the main challenges in the wireless delivery of data and Internet is providing quality service. QoS (quality of service) is also linked to the ability of

Corresponding author: Slava Yordanova, Ph.D., assoc. professor, research fields: computer and communication systems and technologies. service providers to adapt their networks for transmission of voice (Fig. 1).

To properly analyze the possibilities of using Vo802.11 as an alternative voice communication, it is necessary to analyze potential weaknesses of the network in order to minimize or eliminate them. Functions and applications of network 1 largely depend on signalization. With the use of modern VoIP protocols and good programming of new functions, variety of applications satisfying the ultimate user are achieved.

\section{Experimental Results}

To provide quality speech comparable to the PSTN, it is necessary to minimize delays, jitter and packet loss in Vo802.11 networks. Regulating QoS to the wireless broadband network is accompanied by additional network requirements.

To measure the voice quality, two tests for ensuring metric are currently available: MOS (mean opinion score) and PSQM (perceptual speech quality measurement) (Fig. 2).

Investigation on IP traffic and signaling tests which were made, were carried out under the scheme of Fig. 3.

Two simultaneous calls from endpoint 192.168.1.3 to 192.168.1.4 were utilized and the results were seen within two minutes. 

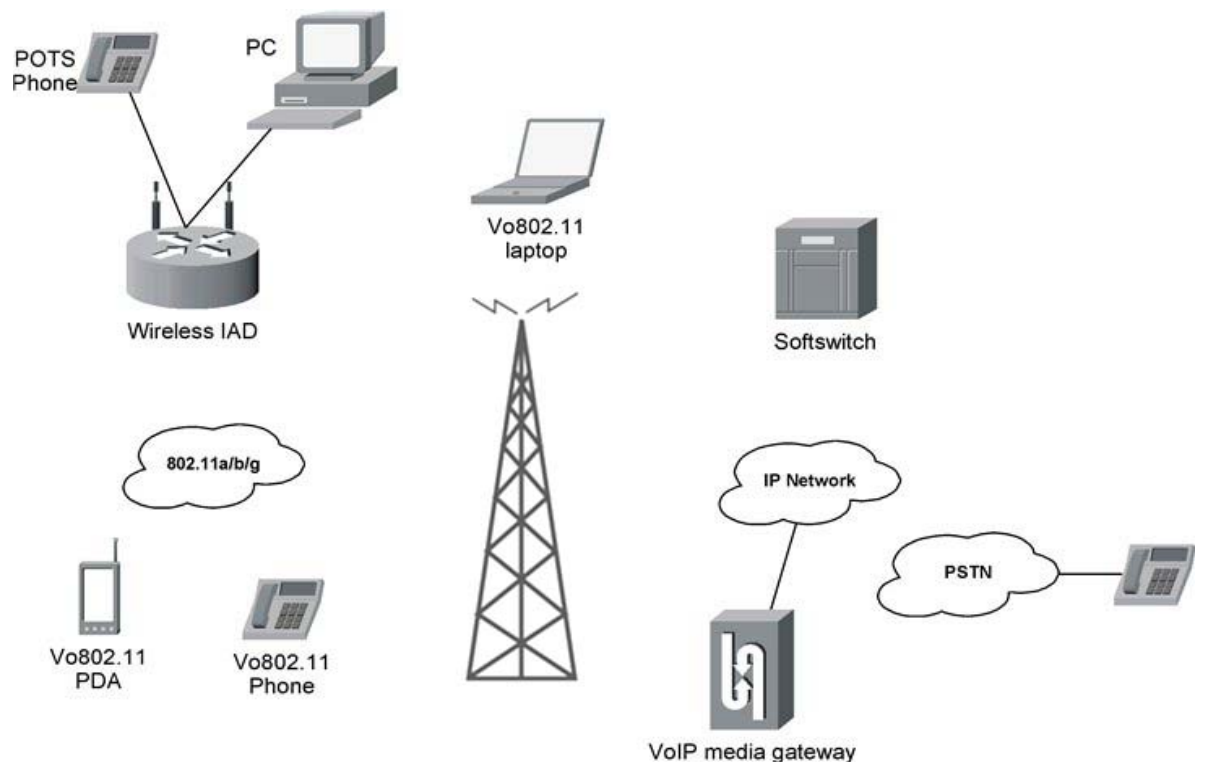

Fig. 1 Broadband network for speech transmission.

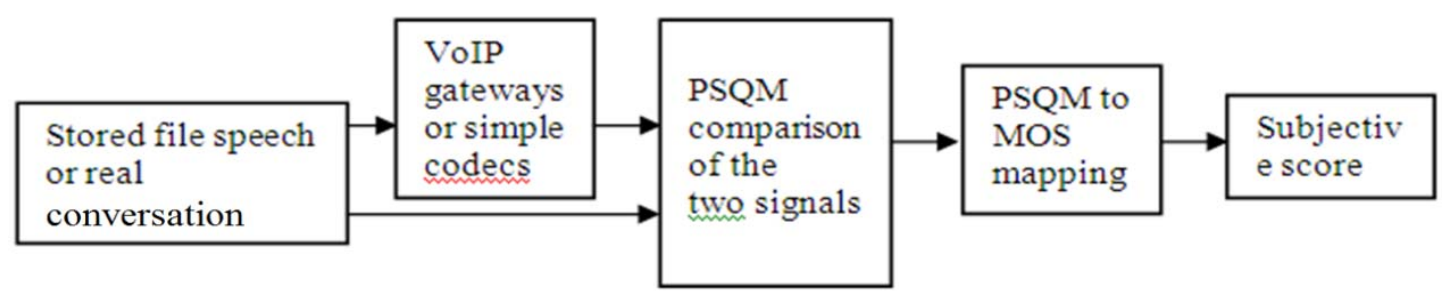

Fig. 2 A process of PSQM [2].
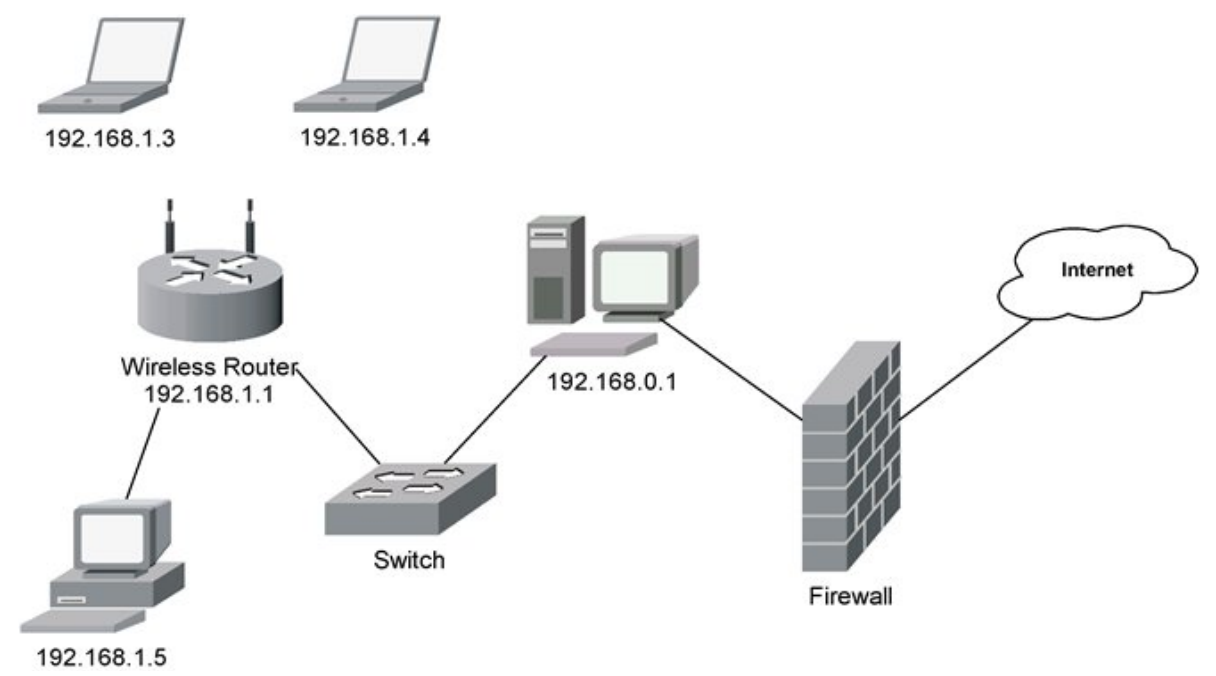

Fig. 3 Network configuration.

The minimum, maximum and average values of some of the most important parameters such as jitter, packet intervals and used bandwidth are given in Table 1.

The addresses of the source and recipient along with their ports and the type of used media are given in Table 2.

The results in Table 3 refer to indicators of monitored calls [3].

RFactor/MOS Score are characteristics of the speech 
Table 1 Audio details.

\begin{tabular}{llll}
\hline Metric & Average & Low & High \\
\hline Src Jitter (ms) & 0.712 & 0.007 & 3.273 \\
Dest Jitter (ms) & 0.911 & 0.005 & 2.735 \\
Src Packet Interval (ms) & 2.431 & 0.009 & 45.001 \\
Dest Packet Interval (ms) & 2.561 & 0.110 & 33.792 \\
Src Bandwidth (kb/s) & 127.691 & 127.421 & 133.333 \\
Dest Bandiwdth (kb/s) & 127.654 & 127.405 & 137.143 \\
\hline
\end{tabular}

Table 2 Type of used media.

\begin{tabular}{lll}
\hline Parameter & Node A & Node B \\
\hline Address & 192.168 .1 .3 & 192.168 .1 .4 \\
Port & 40000 & 40000 \\
Media Type & G.711 Alaw & G.711 Alaw \\
SSRC & 00294823 & 4AE13D6C \\
Audio/Packet (ms) & 3 & 3 \\
Frames/Packet & 3 & 3 \\
Total Packets & 79834 & 79814 \\
Packets Lost & 0 & 0 \\
Longest Packet Loss Burst & 20 & 13 \\
Total Payload Bytes & 1916016 & 1915536 \\
\hline
\end{tabular}

Table 3 Audio QoS.

\begin{tabular}{lll}
\hline Metric & Source & Destination \\
\hline Listening R Factor & 93 & 93 \\
Conversational R Factor & 93 & 93 \\
Listening MOS Score & 4.195 & 4.195 \\
Conversational MOS Score & 4.195 & 4.195 \\
P.862 Raw MOS Score & 4.449 & 4.449 \\
Burst Packet Lost Rate (\%) & $0.000 \%$ & $0.000 \%$ \\
Average Burst Length (ms) & 0 & 0 \\
Average Gap Length (ms) & 28411 & 48546 \\
Good Packets & 40005 & 40010 \\
Lost Packets & 0 & 1 \\
Discarded Packets & 2 & 0 \\
Media Type & G.711 Alaw & G.711 Alaw \\
\hline
\end{tabular}

tract for each individual media stream and are assessed subjectively. The software used in this research processes models including time-varying characteristics such as: sequence of lost packets, discarded packets due to high jitter and others.

Burst Packet Loss Rate gives the number of the lost packets in percentages in case of unusual circumstances.

Average Burst Length shows the average duration in milliseconds of the unusual circumstances that would lead to packet loss.
Average Gap Length is the average length of the intervals in milliseconds in the event of unusual circumstances in the respective media streams.

Good Packets is the number of received packets at the two endpoints.

Lost Packets is the number of lost packets in the network for both endpoints.

Discarded Packets are the packages that get rejected when the delay is exceeded or earlier arrival at the source or recipient has happened.

Media Type shows the type of media format used.

A graphic expression of the quality of the audio signal is shown in Fig. 4. QoS reports are given, as a result of analysis of VoIP calls over 30 seconds.

The parameters of the audio stream in graphical form are presented in Fig. 5. They are the number of packets per second, jitter in the audio channel, packet interval of audio packages and the speed used in kbps.

The ranges of delays of the individual packets during transmission of G.711 audio stream have network delay below $10 \mathrm{~ms}$, and the greatest delays do not exceed 60-70 ms with limit values $150 \mathrm{~ms}$ in one direction (Fig. 6).

The majority of packages have jitter below $3 \mathrm{~ms}$, while the largest values do not exceed 8-10 ms, which again is within the norm of $20 \mathrm{~ms}$ (Fig. 7).

G.722.1: The coding system uses SB-ADPCM (subband adaptive differential pulse-code modulation) at the speed of $64 \mathrm{kbps}$. The system has three modes of operation for $7 \mathrm{kHz}$ audio encoding, corresponding to the bit rate which is 64 or 56 , or 48 kbps. For the study a speed mode of $64 \mathrm{kbps}$ is used.

G.723.1 6.3 kbps: This recommendation of the ITU has two standard speeds -5.3 and 6.3 kbps. The higher speed, which is used in the study gives better quality. The length of the voice frame is $30 \mathrm{~ms}$ and further prediction of $7.5 \mathrm{~ms}$ allows algorithmic delay of 37.5 ms. Encoder is designed to compress a voice with good quality with reduced complexity and it is not suitable for music [4]. 
Call Summary $\mid$ Call Flow $\mid$ Call Trace $\mid$ Call Metrics $\mid$ Audio Summary $\mid$ Audio Details Audio QoS $\mid$ Video Summary $\mid$ Video Details $\mid$ RTCP $\mathrm{S}$
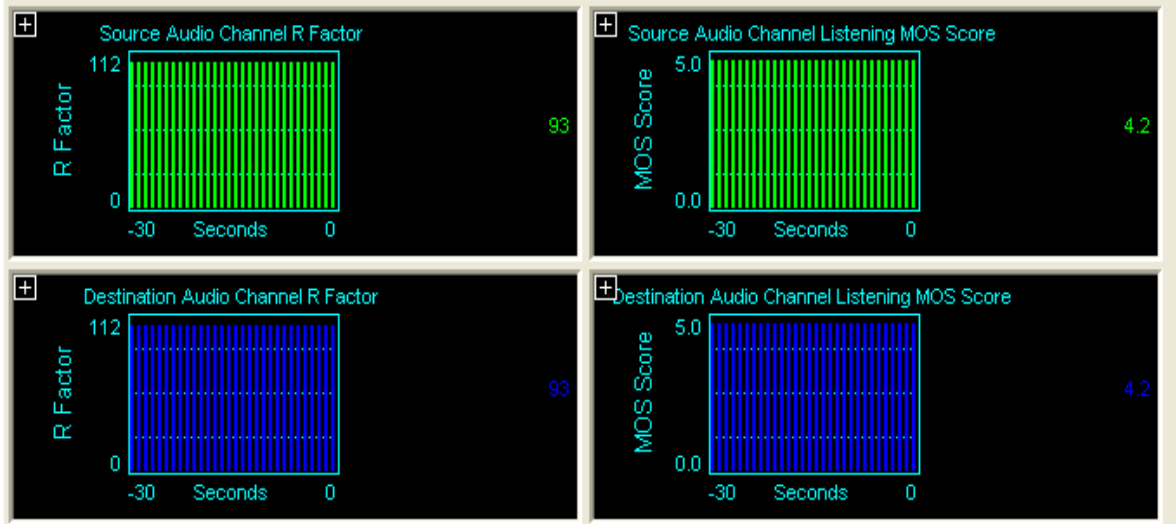

Fig. 4 Audio QoS.

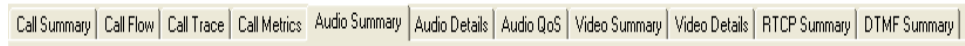
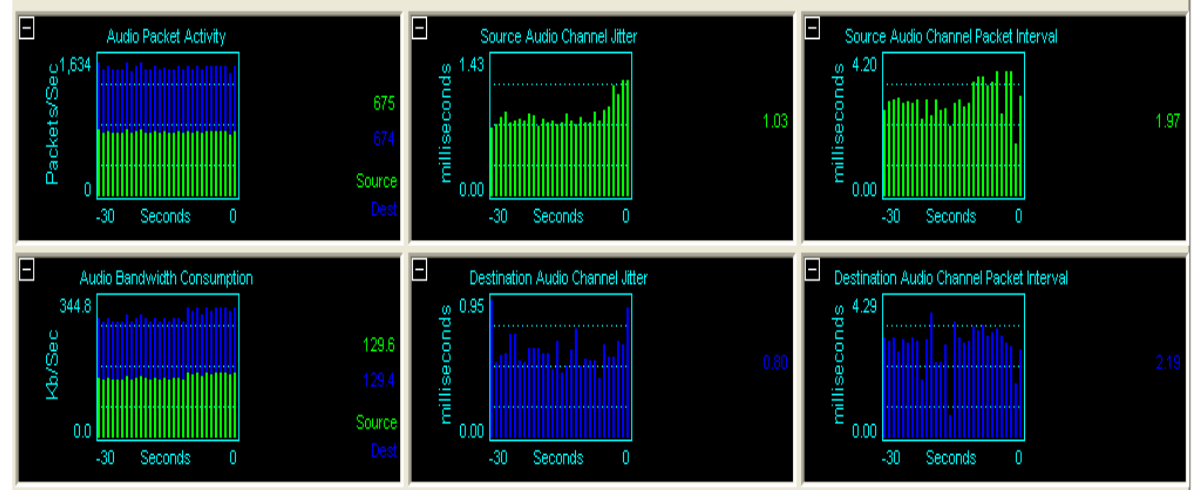

Fig. 5 Graphic form of the audio stream parameters.

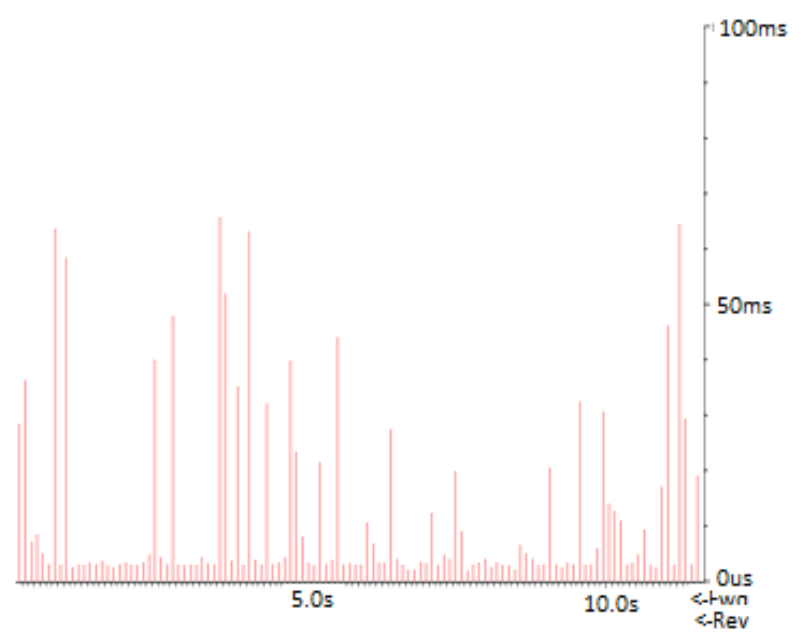

reverse direction of the conversation are fully comparable with the requirements.

Data on network delays of packets, jitter, and packet

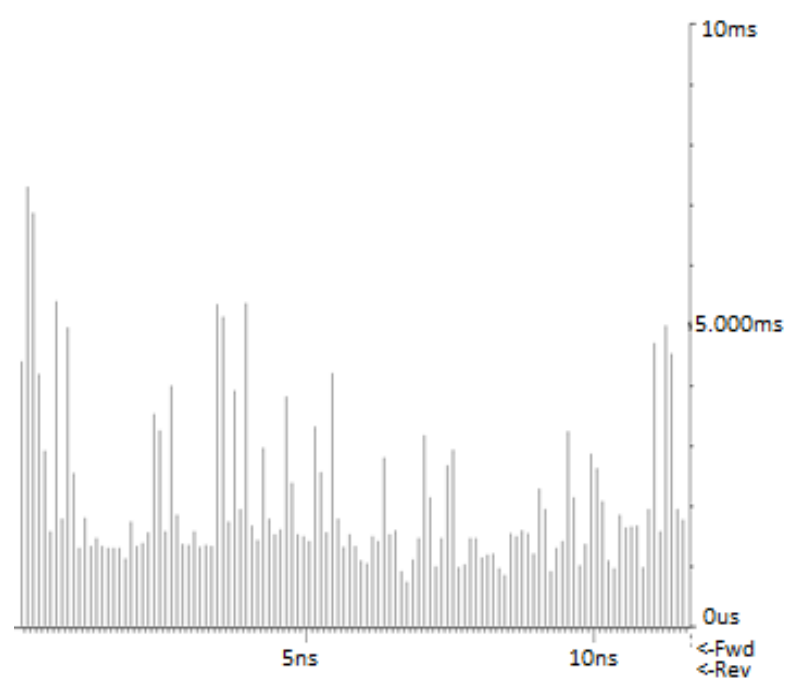

Fig. 7 Jitter of the individual packages. 


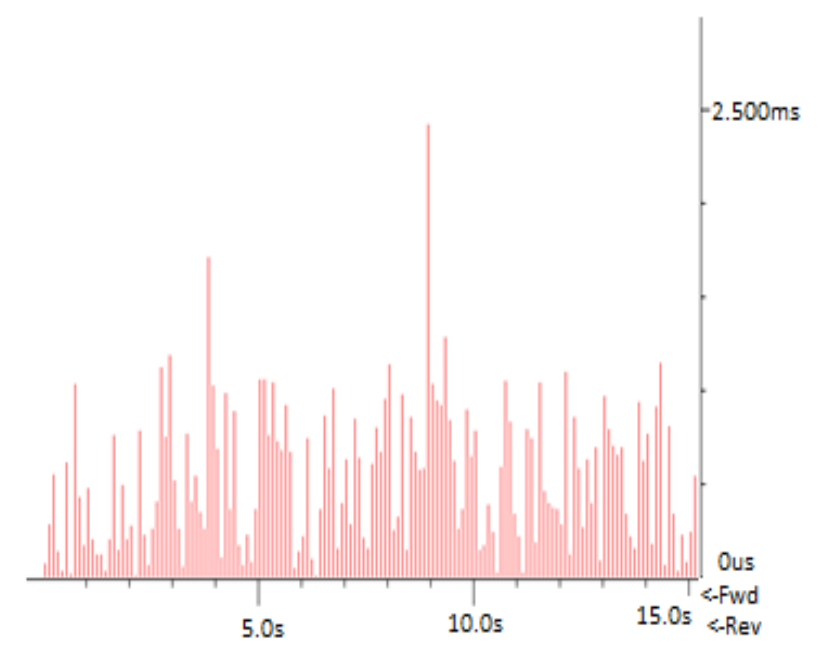

Fig. 8 Delays of packets in G.723.1 6.3 kbps.

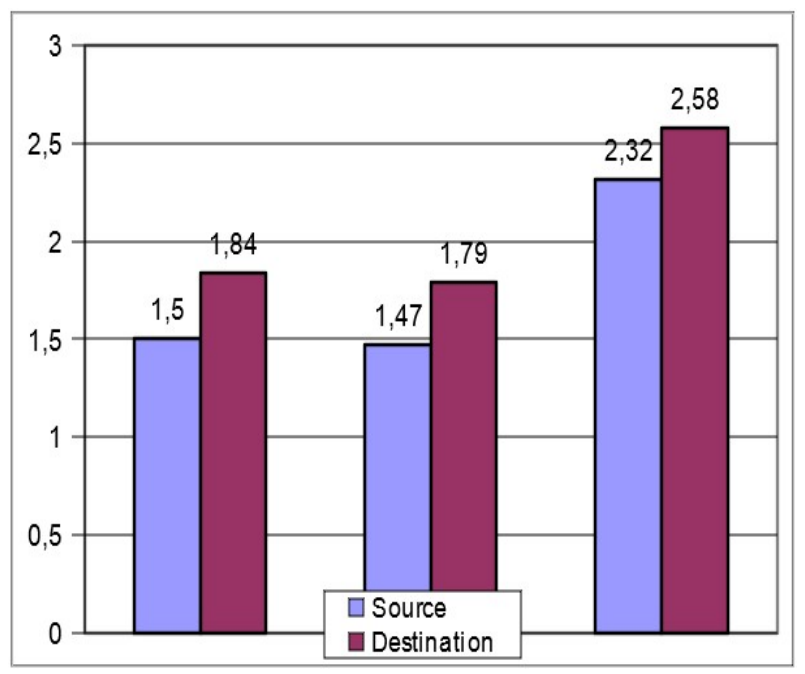

Fig. 9 MOS results in loaded wireless network.

intervals was obtained for real SIP call. The calls have good quality-understandable, without echo and without noise.

\section{Conclusion}

The testing of the realised Vo802.11 network shows its ability to compete PSTN under comparable conditions: the use of uncompressed voice and the protocols that are appropriate for these conditions. The possibility of using compressed formats of the media stream while maintaining the best operational parameters provides sizeable potential for the system and makes it flexible for network designers and the advanced signaling provides high intelligence of the entire network. These factors make it possible to quickly build stable and reliable systems providing quality services to the end users.

In the process it became clear that the disadvantages associated with the range of wireless networks and their security cannot be a real obstacle to the implementation of Vo802.11 voice transmission in real time.

The main contradictions that still remain to the fore are those related to QoS. To show the behavior of a system Vo802.11 in practice, in real conditions, a wireless tract was examined for two major signaling protocols SIP and H.323, and for the only transport protocol that was possible at the moment-RTP. The obtained results and the comparisons show the joint work of SIP and H.323 — SIP realizing a signaling over packet switched networks, and H.323 building interface via gateways to the PSTN.

Taking into account the obtained data concerning different aspects of the behavior of the investigated system and recognizing that no special measures for providing better QoS, such as RSVP and DiffServ, were used, we can say that the obtained results can guarantee the operability of Vo802.11 for voice communications.

\section{References}

[1] Wallingford, T. 2005. Switching to VoIP. O’Reilly \& Associates, Inc.

[2] Agarwal, A. 2000. "Quality of Service (QoS) in the New Public Network Architecture.” IEEE Canadian Review.

[3] Cisco Systems. "Resource Reservation Protocol." http://www.cisco.com (Access Sept. 1, 2016).

[4] Ohrtman, F. Softswitch: Architecture for VoIP. New York: McGraw-Hill. 